\title{
Is the United States Preventive Services Task Force still a voice of caution?
}

In this article (BMJ 2017;356:j743, doi:10.1136/bmj.j743) we incorrectly stated that the Kaiser and Duke evidence based practice centers (EPCs) receive funding from industry. In fact, Kaiser Research Affiliates EPC does not receive industry funding. It is the parent organisation, Kaiser Permanente, which receives $10 \%$ of its funding from industry; the Duke EPC is housed within the Duke Clinical Research Institute, which receives $63.3 \%$ of its $\$ 331 \mathrm{~m}$ annual revenue from industry. 